\title{
Src Expression during Murine Development
}

\author{
Shur-Tzu Chen ${ }^{1}$, Shu-Mien Chuang ${ }^{2}$ and Jiang-Ping Wang ${ }^{2}$ \\ Departments of Anatomyl and Biology ${ }^{2}$, National Cheng Kung University, Taiwan, R. O. C. \\ (Received 14 September 1999; and accepted 4 November 1999)
}

\begin{abstract}
The study examines the distribution of $s r c$ gene products in mouse embryo tissues, to reveal the patterns of expression that may elucidate the potential role of the Src family during development. Immunohistochemical analysis demonstrates that eight members (c-Src, Fyn, Yes, Lyn, Lck, Hck, Fgr and Blk) within the Src family are expressed early in dividing cells from preimplantation stages and remain in many tissues until the late prenatal stages. Experimental results indicate that Src expression is present prevalently in fetal development in tissues from all three germ layers. For instance, ectodermal derivatives that displayed Src expression included both nervous system and epidermis cells. Meanwhile, mesodermal tissues including chrondrocytes, kidney, gonad, and endoderm-derived tissues, such as from the heart, lung and intestine, also expressed Src immunoreactivity. In the heart, Src is expressed at embryonic day 8 , the time when myocardial contraction begins, and remains throughout the embryonic stage. However, widespread expression becomes restricted to certain cell types with maturation. For example, the Src family is restricted to the epithelial lining and muscular layer in the gastrointestinal ducts during the later stage of embryo growth. This study have observed a slight difference in the progression of the expression and in staining intensity although the overall pattern for the eight Src family members is similar. The wide distribution of the Src family in the developing mouse tissues implies that many immature cells require these gene products for signal transduction and possibly for regulating cell survival.
\end{abstract}

The Src family of protein-tyrosine kinase contains many members (c-Src, Fyn, Yes, Lyn, Lck, Hck, Fgr and Blk), which have been shown to derive from distinct cellular genes that generate proteins competent capable of oncogenic cellular transformation $(3,7,15)$ and primarily functioning in cellular signaling pathways $(8,26)$. The major $s r c$ gene encoded protein in $\mathrm{pp} 60^{\mathrm{Src}}(\mathrm{C}-\mathrm{Src})$ is largely expressed in neuronal tissues and platelets. This fact has led researchers to suggest that c-Src influences neuronal differentiation and maintenance

Correspondence to: Dr J. P. Wang at the above address.

Fax: +886-6-2093007

E-mail: wangcp@mail.ncku.edu.tw of mature neuronal cell function and platelet homeostasis $(4,10)$. The origin and general expression of other Src members have shown in some previous studies. For instance, human c-yes cDNA was originally cloned from a cDNA library derived from cultured human embryo fibroblasts (25), while $l y n$ gene was initially cloned from a placental cDNA library by using a $v$-yes probe (29). Immune complex kinase assays of platelets, $T$ cells and macrophages revealed relatively high levels of Yes and Lyn (9) while Lyn deficient mice develop autoimmune disease (12). The major translational product of the human $l c k$ gene, a lymphocyte-specific tyrosine, is designated pp56 $6^{\text {lck }}$. A related investigation has demonstrated that $l c k$ gene is localized to a site in 
the genome subject to chromosomal abnormalities in lymphomas and neuroblastoma (20). The Lck expression accompanies the appearance of lymphoid cells in the developing human thymus and thus, the Lck expression is considered a marker for cells of lymphocyte lineage (21).

Although the normal function of the Src family remains unclear, its members are not only structurally similar but also share important regulatory mechanisms with membrane-associated tyrosine protein kinase (2). For instance, Hck and Fgr are largely coexpressed and coregulated in hematopoietic cells (17). Several studies have also shown that the Src family is spread through the tissues and organs of various species. The expression of c-Src, Fyn and Yes is ubiquitous, although some members of the family are restricted in their expression, for example being expressed only in cells of the hematopoietic system $(1,2,23,24)$. Moreover, the Src family is distributed widely in developing tissues such as Drosophila embryo and eye, avian embryos, Xenopus laevis and rat spermatogenesis, and human embryonic tissues. The most common expression of the prototype member in this gene family, i. e. c-Src, is observed in developing platelets, heart and neuronal tissues $(16,18,19$, 22 ), while Lyn is expressed largely in neuroblastoma cell lines, macrophages, platelets and $\mathrm{B}$ lymphocytes, and regulates thymocyte development and murine eggs (30). Based on their associations with specific cell surface receptors, researchers have long realized that members of the Src gene family are critical in various signal transduction pathways. The transforming and developmental effects of activated alleles of Src also led to the expectation that Src would be essential in cell growth or differentiation.

This study used immunohistochemistry to examine the expression of eight members of the Src family in mouse embryos. The Src family was found in many tissues from the cell-divided preimplantation to later fetal stages, but particularly high levels were found in heart, nervous and muscular system tissues. According to our results, an early appearance, high concentration and a wide distribution of individual members of the Src family in the nervous system may reveal that these gene products profoundly influence neuronal differentiation and maturation (27). Although the Src family displays similar structures, functions and expressions in murine tissues for Src family, this study found difference in distribution and staining intensity between Src members in various organs, and especially in the non-neuronal part. This observation suggests that the Src family may be important in processes other than cellular differentiation, organogenesis and maturation, and that the members of the family may function differently to previous assumed.

\section{MATERIALS AND METHODS}

ICR strain albino mice were purchased from the Animal Center of National Cheng Kung University Medical College. Females were superovulated with pregnant mare serum and human chorionic gonadotropin (Sigma). Mating was performed overnight in our own facilities. The morning on which vaginal plugs were observed was considered to be embryonic day (E) 0 . The next $24 \mathrm{~h}$ is $\mathrm{E} 1$, and so on. A total of 70 pregnant mice were used and embryos aged from E0 (2-cells onward) to E20. Before streak stage, the embryos were collected containing maternal oviduct or uterus. Meanwhile, the embryos aged from E9 onwards were dissected from uterus and, then, all embryos were washed briefly in phosphate buffer saline (PBS).

Embryos were fixed in $4 \%(\mathrm{w} / \mathrm{v})$ paraformaldehyde in $0.1 \mathrm{M}$ phosphate buffer at $4^{\circ} \mathrm{C}$ for $2-6 \mathrm{~h}$. The tissues were dehydrated and embedded in paraffin. Embryos were sectioned parasagittally and coronally and the sections were incubated for 2 days at $4^{\circ} \mathrm{C}$ with a rabbit polyclonal antiserum raised against c-Src, Fyn, Yes, Lyn, Lck, Hck, Fgr, and Blk (Santa Cruz, diluted to $1: 600$ ). These sections were then washed with PBS and incubated in biotinylated anti-rabbit IgG sera (Vector) for 1 to $2 \mathrm{~h}$. The tissues were washed again and incubated in avidin-biotin complex solution for another 1 to $2 \mathrm{~h}$ period at room temperature, and finally processed for peroxidase reaction and developed according to the glucose oxidase-nickel-DAB method $(5,6,27)$. Control sections were reacted with the primary antibody omitted or replaced by non-immune rabbit or normal goat serum. No positive immunoreactivity was observed in control sections. Some of the sections were stained with hematoxylin and eosin solution for studying the cytoarchitectonics of the developing organs or tissues. On the other hand, the specificity of immunoreaction has been verified, indicating that all antibodies of Src members are non-cross-reacted with other Src family 
tyrosine kinase. For instance, the polyclonal antibody c-Src is produced against the amino acids 3-18 mapping at the amino terminus of Src p60 of human origin (Santa Cruz Biotech). Fyn is raised against a peptide corresponding to amino acids 29-48 mapping with the amino terminal of Fyn p59 of human origin and do not cross-react with other Src family. Lyn is raised against the amino acids 44-63 mapping within the amino terminal domain of human Lyn p56/p53 and non cross-reactive with other tyrosine kinase. Fgr recognizes the residues 47-66 mapping within the amino terminal domain of the human $c-f g r$ gene product. Blk is raised against a peptide corresponding to amino acids 21-43 mapping within the amino terminal domain of Blk of mouse origin. Fgr and Blk are also found not to crossreact with other Src family.

\section{RESULTS}

The tissues studied expressed Src family immunoreactivity (all 8 proteins) from the 2-cell stage or early blastomeres, the earliest point examined. The Src family members were expressed at all development stages, from the preimplantation stages to the late prenatal period. Closely examining the embryo sections under high magnification revealed that the immunostaining appeared throughout most cells over both the nucleus and cytoplasm. The largest and earliest Src expression in the organogenesis was found in the developing heart and in the immature neural tissue of the brain at E8. After about 4 to 7 days, high levels of the Src gene family were visible in the somites (future muscular or skeletal systems), digestive, respiratory, excretory and reproductive systems. Src family expression in most of these organs declined at the end of the fetal stage. The following texts are based on a particular protein (with clear and typical immunostaining) that was chosen to represent each instance at a certain developing stage and in a certain area.

\section{Distribution of Src gene proteins in pre- and post-implantation stages}

The first cleavages of fertilized oocyte appeared in the ampulla of the oviduct about $30-40 \mathrm{~h}$ after fertilization. The embryo continued dividing, as it moved into the uterus between the second and third day. The fourth cleavage, about $60 \mathrm{~h}$ after fertilization, produced a solid ball of morula. Four days after fertilization (E4), the blastula was formed. Slight to moderate Src immunoreactivity was observable as early as the 2-cell stage although the staining intensity remained inconsistent between all members. For instance, masses of immunostained cytoplasm in these blastomeres were present in the Fyn, Lyn, Yes, Lck and Hck-reacted embryo sections while only a few immunoreactive granules for c-Src, Fgr and Blk were in the embryonic cells before the blastulae stages (Fig. 1A-E). By E4, Src family expression was present in the inner cell mass, distal endoderm and trophoectoderm of the blastocysts (Fig. 1E). In this period, Fyn- and Lynimmunoreactivity showed the highest levels among the other members of the Src family.

By E5, the embryo was divided into two regions, a dorsal region with high columnar cells with elongated nuclei and a ventral region with smaller cells with rounded nuclei. The dorsal region created the extra-embryonic structures (such as membranes), and the ventral developed various structures of the embryo proper (Fig. 1F). At this stage, immunoreactivity was more obvious in the endoderm than the ectoderm (Fig. 1F, G). By E6 and E7 the mesoderm appeared as scattered mesenchyme cells between the primitive streak and the proximal endoderm. During these periods, the Src family was present at both endoderm and ectoderm, and the entire family appeared in the mesoderm later, despite Hck immunoreactivity being absent in the ectoderm (Fig. 1G-I).

\section{Src family expression in organogenesis}

At about 8 days gestation, the embryo displayed signs of organ differentiation. Meanwhile, the immunoreactivity of the Src family was discernible in several organs and systems derived from different germ layers. The general distribution and staining intensity of Src family in organogenesis are described below and summarized in Table 1.

First, the mouse circulatory system exhibited a paired cardiac mesenchymal primordium and a crescent-shaped peritoneal cavity appeared from E7, followed by the tubular portion of the heart and aortic arch. As mentioned above, the heart was the first organ to become immunoreactive for most of the Src family, at about E8. The moderate to intense staining was spread in the cardiac 


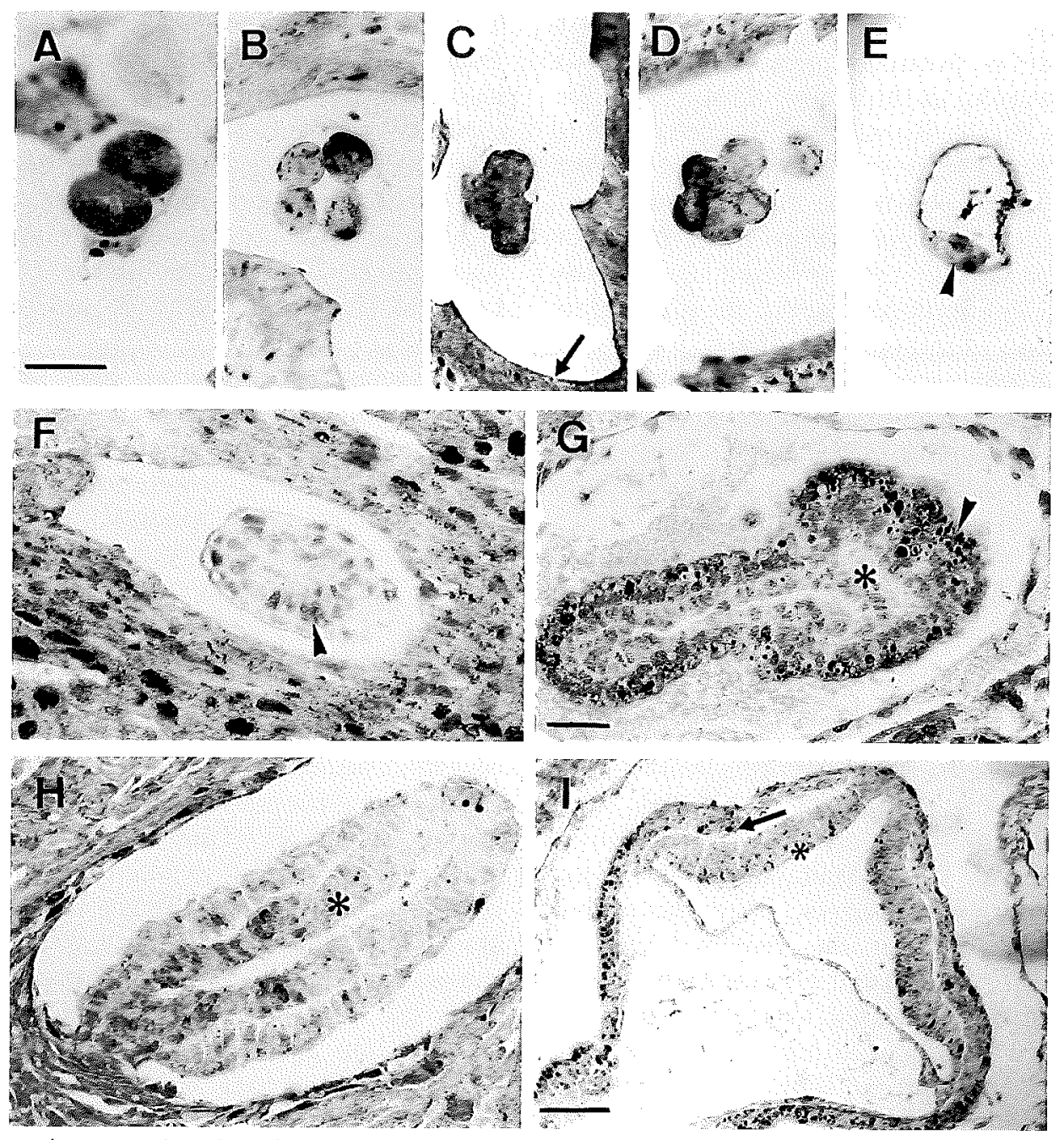

Fig. 1 Photomicrographs showing the expression of Src family during early development. Moderate to strong immunoreactivity for Fyn is observed in a 2-cell (A), 4-cell (B), 8-cell embryo (C), morula (D) and blastocyst (E). Note that the staining cells are observed in the inner cell mass of the blastocyst (arrowhead, E). The maternal uterus is also immunostained (arrow, C). At E5 (F) and E6 (G), Yes-positive cells are distributed mainly in the developing endoderm (arrowhead) while the staining is weak in the ectoderm (asterisk, same in $\mathrm{H}$ and I). The immunoreactivity intensifies later in the ectoderm as Lck expression at E6 (H) and E7 (I) shows. Note that the mesoderm-derived cells are obvious and immunoreacted (arrow in I). The scale bar in A is for all figures except $\mathrm{G}$ and $\mathrm{I}$. Bar $=30 \mu \mathrm{m}$.

muscle cells and their processes in the developing atrium and ventricle (Fig. 2A, B), and was present throughout the prenatal period. During the later stage, however, the immunoreactive cells became restricted in the innermost part of ventricle in some Src members, resembling autonomic nerve bundles (Fig. 2C). The endothelial cells of newly formed large arteries and veins were also immunostained heavily (Fig. 2D).

By E8 the pharyngeal pouch was formed and inductions of laryngo-tracheal groove appeared, which extended into a pair of lung buds at E10. The lung bud branched repeatedly to create right and left lungs and successive levels of the bronchial tree. Around E12, the Src gene proteins were more highly expressed in the thicker and more proximal region of the lung bud than in the thinner distal epithelium (Fig. 2D, E). From E14 onward, Src expression was highly evident in the tracheal and bronchiolar cells of airways but much less evident in the alveoli and pulmonary blood vessels (Fig. 2F). 
Table 1 Summary of the Src family (all 8 proteins) positive cells during murine embryogenesis

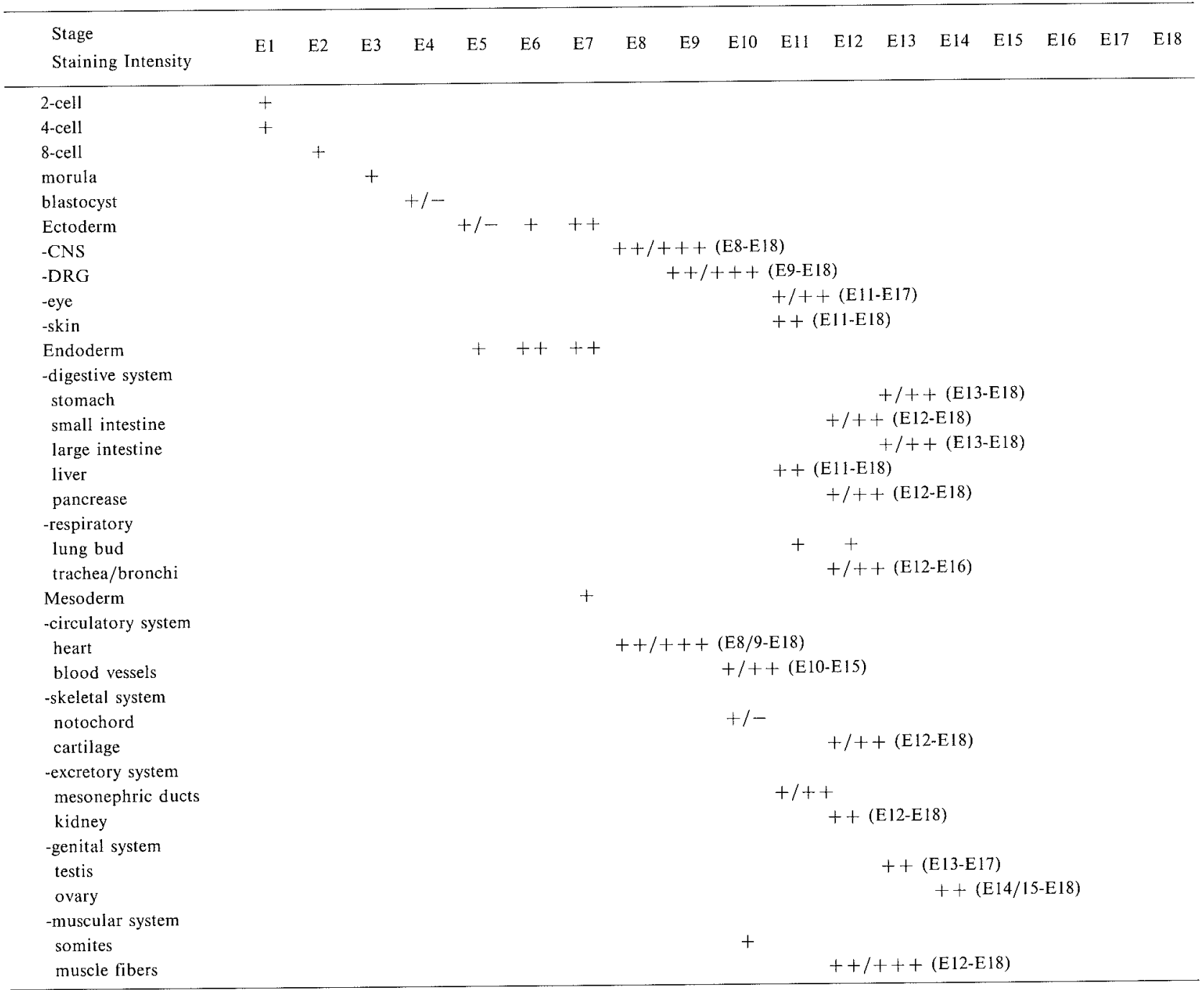

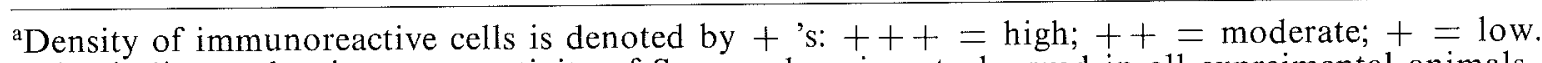
$+/-$ indicates that immunoreactivity of Src members is not observed in all expreimental animals.

The digestive system including the foregut, liver primordium, midgut and hindgut formed as carly as E7.5. In this study, at E9 early expression of the Src family was detected in the epithelium of the developing esophagus, and the small and large intestines, derived from fore-, mid- and hindgut, respectively. Generally, the highest levels of Src expression appeared in the mucosa layer in the middle developing stages, around E12 to E16 (Fig. 2G, I, J). Additionally, a row of parasympathetic nerves, resembling Auerbach's plexuses, was observed in the muscular layer of the developing intestine indicated by Lyn immunoreactivity in an E14 fetus (Fig. 2G). From E16 onward, the epithelium became convoluted with developing villi and all epithelial cells continued to express Src gene proteins. Moderate immunoreactivity was observed in the outer muscular layer in the digestive tube but not in the submucosa, however, the staining strength gradually decreased after E16 or E17.

The liver was derived from the caudal forget tissue at E9 and was hematopoietic from E12.5 to E18.5. Src family expression first appeared in the fetal liver by E11 or one day later (Fig. 2B, D). The Src family was expressed wildly within hematopoietic cells but absent in parenchymal cells. Megakaryocytes which exhibit high levels of Src proteins were also identifiable under higher magnification (Fig. $2 \mathrm{H}$ ). The density and staining 


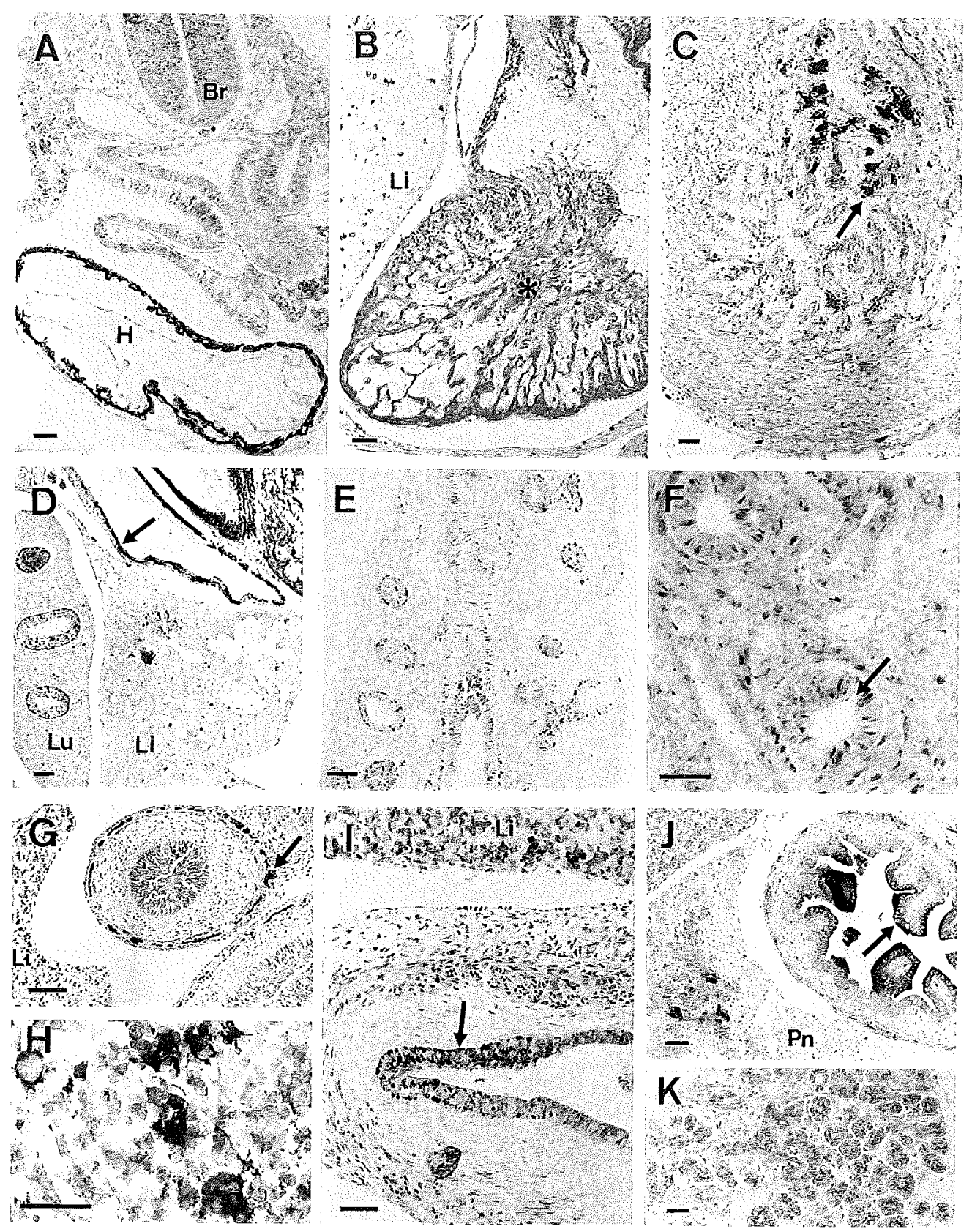

Fig. 2 Src family immunoreactivites in the developing circutatory, respiratory and digestive systems.

(A) shows Lyn expression in the hind brain $(\mathrm{Br})$ and heart $(\mathrm{H})$ at E9. The staining intensity in the heart remains very high by E 12 (asterisk in B), but reduces after E 15 (C). Nerve bundle-like profiles are discernable in the core part of the atrium in an E15 fetus. (D) shows moderate to strong immunoreactivity for Lck in the dorsal aorta (arrow), lung (Lu) and liver (Li) of an E12 fetal section. At E14, Lck-positive cells are spread around the bronchial tree $(\mathrm{E})$. Under higher magnification $(\mathrm{F})$, the positive cells are primarily located in the epithelium of the bronchi (arrow). (G) shows Lyn-immunoreacted liver and intestine by E14. Note a very dark reaction detected in the muscular layer of intestine, resembling Auerbach's nerve plexus (arrow). A high level of immunoreactivity for $\mathrm{Fgr}$ is also observed in the liver cells $(\mathrm{H})$. Meanwhile, Yes expression is present in both the liver and the stomach (I). Notably, the darkest reactivity is in the mucosa layer (arrow, same in J). Similarly, c-Src immunoreactivity is expressed in the innermost layer of the intestine by E17 (J). At this stage, c-Src expression in the pancrease (Pn) is obvious $(\mathrm{J}, \mathrm{K})$. Bar $=30 \mu \mathrm{m}$. 
intensity of immunoreacted cells remained prominent at the later period. The exocrine pancreas was formed by two buds originating from the endodermal lining of the duodenum. By E12 the dorsal and ventral pancreatic rudiments fused, and then the endocrine cells migrated from the duct system and aggregated around capillaries to form isolated clumps of cells, known as the islets of Langerhans. Our results indicated high immunoreactivity in the clumps of endocrine secretory cells alone with little Src family expression in the pancreatic acini or duct between E13 and E18 (Fig. 2J, K).

Salivary glands, $i$. $e$. the parotid, submandibular and sublingual glands began as solid epithelial proliferations or buds from the primitive oral cavity and then grew into the underlying mesenchyme around E9. The buds of the parotid glands appeared first from the oral ectodermal lining near the angles of the primitive mouth. The buds of submandibular and sublingual glands appeared later, and then branched, differentiated and canalized to form acini and ducts. This study found that moderately immunoreacted cells tended to be distributed in the base of the acini, connective tissue and ducts in the parotid gland from E13, and in the other two salivary glands at E15 (Fig. 3A).

The thyroid was the first endocrine gland to develop in the embryo at about E11. At E13 although immunoreactivity remains very low, it increased to moderate within 2 or 3 days and became distributed on the enlarged gland cells with early follicles (data not shown). Simultaneously, immunoreacted parathyroid gland could be seen surrounded by the thyroid and located dorsolaterally. The thymus gland, also a lymphoid organ, developed upwards from the epithelium of the $3 \mathrm{rd}$ and 4th pharyngeal pouches and then became infiltrated by lymphocytes derived from the haemopoietic tissue after E12. Figure $3 \mathrm{~B}$ reveals that by $\mathrm{E} 15$, moderately to darkly immunostained cells were present in the various types of thymocytes but not in the epithelium beneath the capsule by E15.

In the excretory system, the nephrogenic cords appeared around E8, while pronephric tubules and collecting pronephric ducts were visible by E10. At E12, each mesonephros consisted of crowded and still undifferentiated nephric units, and metanephric urethral buds sprouted from the posterior part of the nephric duct. At this timepoint, the Src family was expressed moderately on the epithelium of mesonephric and metanephric ducts. In the developing kidney (metanephros), branched ureteric buds were surrounded by metanephric caps and some primitive tubular structures from E13 to E14, and then many elements of the mature kidney were perceivable (Fig. $3 \mathrm{C})$. By E16 or one day after, the collecting ducts and metanephric tubules contained the highest levels of Src family expression, while the cap tissue and glomeruli contained low levels of staining, and there were negative reactions in the cells lining the pelvis or loose mesenchyme of the medulla (Fig. 3D, E). Src immunoreactivity was also observable in the epithelium of the ureter by early at E11, and the outer muscular layer displayed positive staining shortly after. The developing urinary bladder became distinguishable by E12, however, the expression of the Src family in the smooth muscle was low at stages when fetal tissue was compared with that of skeletal muscle (Fig. 3G).

The origin and migration of the primordial germ cells to their final site were the same in both sexes. The primordial germ cells developed very early, migrated to the dorsal mesenteries, and finally closed to the mesonephroi around E10.5. By E13, the genital ridges enlarged considerably in the male with interstitial tissue becoming distinguishable from the sex cords of the testis, and by E14 each testis cord displayed early spermatogenesis. At this time, the dividing spermatogonium in the testis cords (the future seminiferous tubules) exhibited dense immunoreactivity, but interstitial cells did not (Fig. 3C). In the female, the temporary solid oviducts extended towards the cloaca and the germinal epithelium was distinguishable from the internal stroma at about E13. Figure $3 \mathrm{~F}$ shows Fyn immunoreactivity at E15 in an immature ovary, where the staining intensity was higher in the ovigerous cords than in the stroma. Furthermore, no immunoreactivity was found in the degenerating mesonephros, while the oviduct (Mullerian duct) showed positive staining. Although the development of the cortex of adrenal gland from the mesoderm and the differentiation of the medulla from neural crest cells were considered to be early, Src expression was visible at E13. During the later developing period, the high immunoreactivity was observed primarily in the medulla (Fig. 3D).

The primordium of the spleen, which was located just dorsal to the stomach and near the level of 


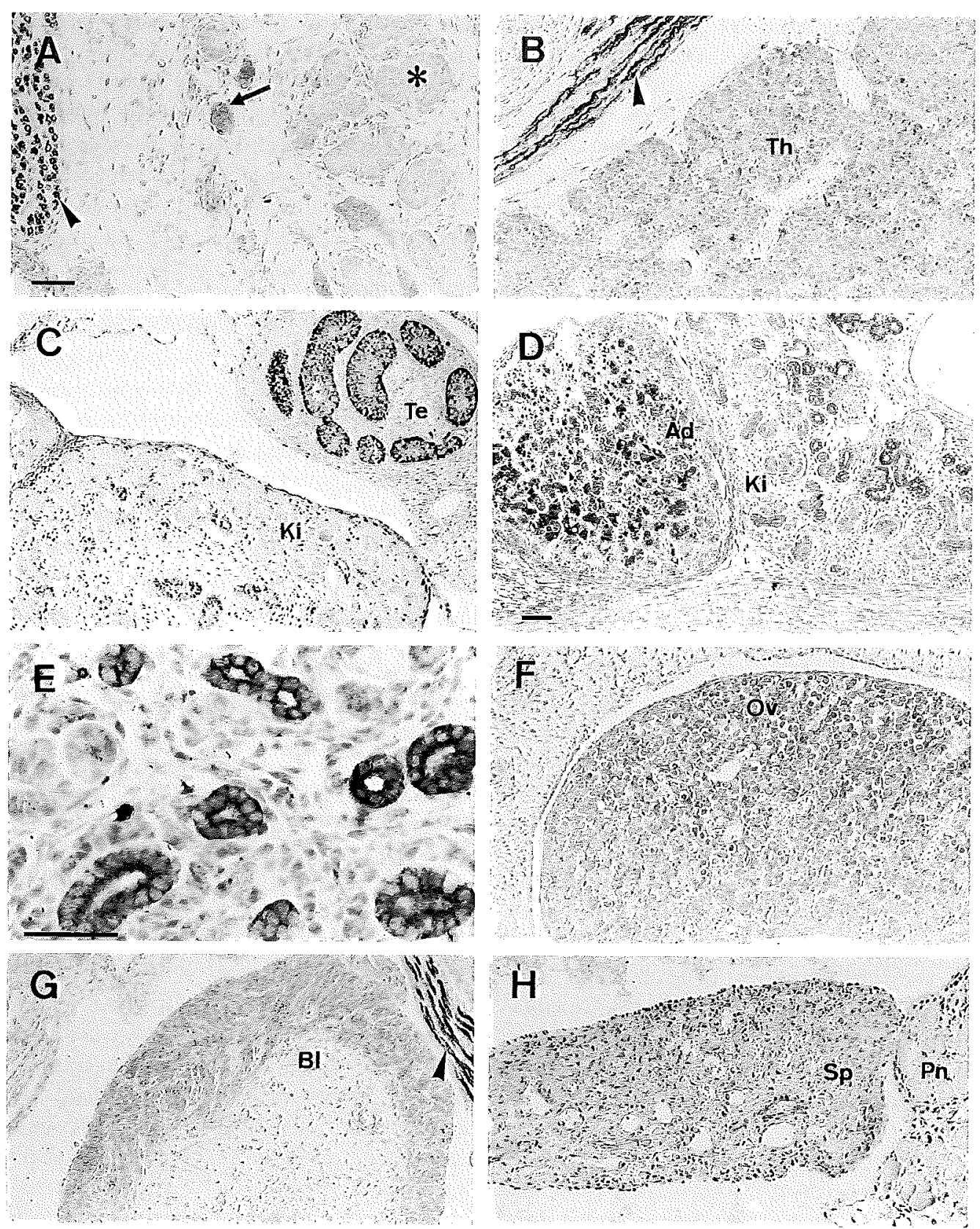

Fig. 3 Microphotographs showing the Src family in the muscular, endocrine and excretory systems as well as in the gonads. By E15, Fgr-positive cells are visible in the developing sublingular/submandibular glands (asterisk, A). Some serous-like cells are immunostained intensely (arrow). (B) shows Fgr immunoreactive thymus (Th) at the same stage. Compared with the staining intensity in other systems, the skeletal muscle fibers exhibit the darkest immunoreactivity as shown in (A), (B) and (G) (arrowheads) before and after E15. (C) shows Hck-containing cells in the left metanephros or definitive kidney (Ki) and testis cord (Te) at E14. The immunoreactivity increases gradually, as indicated an E17 kidney section (D). High magnification (E) shows that the positve cells are concentrated on the collecting tubules of the developing kidney. Meanwhile, the adrenal galnd (Ad) is also immunoreacted (D). Generally, the staining cells are restricted in the neural crest-derived medula part. F: the ovary (Ov) develops slightly later than the testis. By E15, for instance, a moderately stained profile for Fyn is visible. Meanwhile, the Src family is highly expressed in the skeletal and cardiac muscle fibers, but the staining intensity is insufficient in the smooth muscle of the urinary bladder (Bl, $\mathrm{G})$. The spleen $(\mathrm{Sp})$ is also immunoreactive for all Src family at this time $(\mathrm{H})$. The scale bar in A applies to all figures except D and $\mathrm{E}$. Bar $=50 \mu \mathrm{m}$. 
gonad appeared at E13; however, it was not easily identified. From E15 onwards, distinct immunoreactivity could be discerned in the elongating spleen which was not yet differentiated into the typical pulp areas (Fig. 3H).

In the muscular system, mesocardial primordia appeared initially and cardial myoblasts developed later. By E7.5 myotomes arose from somites in the cervical region, by E11 myotomes appeared with longitudinally directed myoblasts with ventral processes in some, and by E14 somite muscle fibrils could be found. The Src family was present in somites at E10 and by E13 high levels of immunoreactivity appeared, centered in the back region of the skeletal muscles. The heavy staining was observed both in the cell bodies and cytoplasm of muscle fibers throughout the body and was expressed to the end of the prenatal stage, as observed in the muscles obtained from the larynx, neck, abdomen and tongue (Figs. 3A, B, G and 4A). As mentioned earlier, Src expression in the smooth muscles, such as in the bladder, was less than that in striated muscles.

The epithelial cells of hair follicles were immunoreactive while the central portion was Srcnegative (Fig. 4B). The staining intensity was obvious at E14 to E17. Besides, Src family expression was also observable in the developing limb. Interestingly, Src immunoreactivity was higher the interdigital zone cells but lower or even absent in the apical and digitial zones by E13, while the positive cells were later clustered around the epidermis (Fig. 4C). As the digits separated between E14 and E15, immunoreactivity was also present in the precartilaginous mesenchymal condensations surrounding cartilage plates and long bones of the limb (Fig. 4C). However, the Src family shifted its expression to the outer border of the ossific vertebrate by E17 (Fig. 4D). Additionally, the ectoderm-derived lens was identified early at E12 and the Src family was expressed later. For instance, the high levels of c-Src immunoreactive cells appeared in lens epithelial cells by E15 (Fig. 4E), while 2 or 3 days later the staining intensity reduced.

High density of Src family immunoreactivities in the neural crest-derived non-neuronal organs

Adrenal gland. The expression of Src family in adrenal medulla and other organs originating from the neural crest was interesting. This study observed expression of the Src family in the inner medulla cells of the adrenal gland, but not in the outer cortex (Fig. 3D). Moderately immunoreacted cells appeared at E14 and the staining intensity and number of positive cells increased as the organ developed.

Pigment cells. As well known, melanocytes originate in the neuroectoderm or neural crest and during development migrate to the epidermis where they spread through the basal layers. This investigation first observed Src family expression on a simple squamous epithelium of skin by E13. The intensity and number of immunoreacted cells also increased towards the end of the prenatal stage (Fig. 4C). The distribution of Src-positive cells was consistent with the location of melanocytes detected by Nissl stains.

Mesectodermal derivatives. Neural crest cells are unique in that, despite their neuroectodermal origin, they significantly contribute to mesenchyme in the head and many other structures. In the branchial or pharyngeal arches, these cells contribute extensively to the formation of the face, neck, nasal cavity, mouth, larynx and pharynx. Most of the skeletal and connective tissues in the head and neck are derived from neural crest mesenchyme. Herein, slightly immunostaining mesenchyme-like loose tissues were detected early in E11 in the head surrounding the brain. The staining intensity grew in the newly formed skull, meninges and connective tissues of the head (data not shown). Dermis and smooth muscle from the head, as well as connective tissue components from the developing tooth papillae, glands and aortic arch were also immunoreactive for the Src family around E13, and they maintained moderate immunoreactivity until the late fetal stage.

\section{Different expression of Src members during organogenesis}

The changes of Src family expression in a single organ or system have been described above and are summarized in Table 1 . Figures $4 \mathrm{~F}$ to $\mathrm{H}$ illustrate the Src expression at E10, 12 and 16, indicating that the immunoreactivity was altered at different developing stages. Moreover, different members of the Src family slightly differed in terms of staining intensity, distribution and the appearance. Table 2 summarizes the variation of Src members in the non-neuronal systems. 

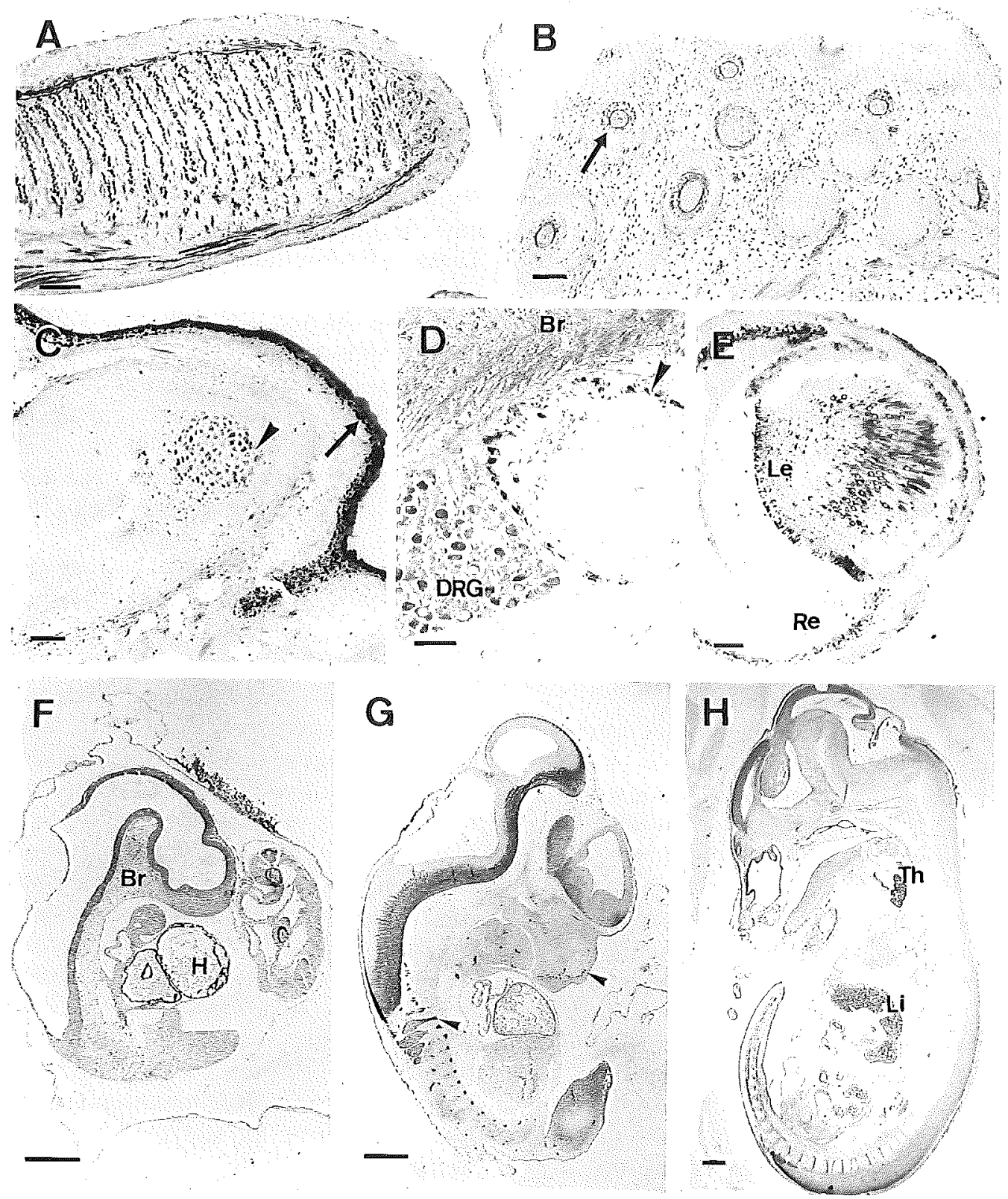

Fig. 4 Src family immunoreactivity in the embyonic organogenesis. As memtioned above, muscle fibers distributed in the E15 tongue are immunostained strongly by Blk antibody (A). The Fyn-positive cells are found in the hair follicles (arrow, B) and the pigmental cells of the skin (arrow in $C$ ) as well as in the cartilage of one fore limb (arrowhead, C) at E15. However, two days later, the staining cells are only detected at the borders, and not the center of the vertebrate (arrowhead, D). c-Src-immunoreactivity appears in the developing lens and retina at E14 (E). The ectoderm-derived lens are stained heavily, but the stains gradually become more faint. Finally, the staining intensity and distribution of Lyn- (E10, F), Fgr- (E12, G) and Hck-(E16, H) immunoreactivity in the parasagittal sections varies between development stages. Notably, the highest expression of brain, spinal cord and heart occurs by E10, while the peripheral nervous fibers (arrowhead) become dark staining by E12. Moreover, the immunoreactivity is most obvious in the thymus and liver at E 16 . Bar $=$ $50 \mu \mathrm{m}$. 
Table 2 Summary of the differences in the reaction and distribution of Src members

$\begin{array}{ll}\text { 2-cell \& 4-cell } & \text { the highest staining intensity for Fyn } \\ \text { 8-cell blastomeres } & \text { Fyn and Lyn showing intense expression } \\ \text { morula \& blastocyst } & \text { high levels for Fyn, Lyn, Hck; moderately for Yes, Lck } \\ \text { E5-E7 } & \text { All Src members present in the endo-, meso- and ectoderm except Hck } \\ \text { E8 } & \text { Fyn, Lyn, Lck, Hck, Fgr highly expression in the heart } \\ \text { E9 } & \text { Yes, Blk, c-Src detected in the heart } \\ \text { E10 onward } & \begin{array}{l}\text { all expressed in the digestive system and mainly located in the mucosa layer; Lyn } \\ \text { observed in the parasympathetic-like nerve fibers }\end{array} \\ \text { E11 onward } & \begin{array}{l}\text { lung bud and bronchi showing Src family; Yes and Hck appearing earlier than } \\ \text { others, Fgr present the latest }\end{array} \\ \text { E12/13 onward } & \begin{array}{l}\text { Fgr and Blk present the collecting ducts, Lyn in nephors, the others expressed both } \\ \text { in the nephros and tubules of the kidney } \\ \text { somites and muscle fibers showing high levels of Src expression except Hck and Blk } \\ \text { E10/11 onward }\end{array}\end{array}$

\section{DISCUSSION}

The investigation has first demonstrated that the src proto-oncogene proteins appear early in the pre-implantation stage, from the 2-cell to blastocyst period, and appear in derivatives of all three germ layers during the middle of the fetal stage. Ectodermal derivatives that contained the Src family included cells of the CNS and PNS, sense organ and epidermis (27). Mesodermal tissues that express Src proteins included chrondrocytes, kidney, gonads and tongue muscle. Meanwhile, liver, epithelium part of lung, intestine and excretory glands, endoderm-derived tissues, also expressed Src family products. However, although Src immunoreactivity is widespread during embryogenesis, it is not ubiquitous. For example, Src is absent in the cortex of the adrenal gland, the mesenchyme of the lung and kidney, and in the loose connective tissue zones, excepting the head and neck.

Interestingly, Src family was most prevalent in the epithelium part of the digestive and respiratory systems as well as in the urinary bladder. The immunoreactivity in these zones appears early but the moderately staining cells are restricted in the endoderm-derived tissues. This pattern indicates that the expression of the Src family is associated with proliferative compartments. Additionally, Src expression appears limited to cells at specific stages of differentiation. For instance, in embryonic skin, Src family are only immunoreactive in the basal cells of newly formed hair follicles while the mesenchymal cells are Src-negative. Corre- spondingly, staining in the epithelium of mesoand metanephric ducts as well as the collecting tubules of the immature kidney from E14 to E17 was strong but in the capsule cells of the most mature glomeruli during the same period staining was weak. These observations imply that Src expression sometimes is associated with morphological transition from undifferentiated stem cells to committed precursor cells, and that the gene proteins are downregulated once these cells have started terminal differentiation.

Another interesting observation is the presence of Src proteins in several components of the developing muscular system, including skeletal, smooth and cardiac muscles. As mentioned earlier and in previous report on chickens (11), Src proteins are expressed most prominently in the primitive heart, when myocardial contractions begins to appear. Src immunoreactivity has also been noted in the newly formed somites and then the staining intensity is upregulated in the myoblasts and muscle fibrils, distributed in the developing tongue, back and limbs. Furthermore, our results indicate very dense immunoreaction in a thin layer of gastrointestinal muscularis propria. The frequency of abundant Src family protein in various types of muscular systems suggest that it may play a role in mediating the differentiation, growth or trophic effects of nerves upon one another. The high levels of Src expression also suggest that Src proteins can be a specific marker for the developing heart and other muscular tissues. Moreover, the high levels may also show that only some organ systems (in particular the 
nervous system and muscle) cause the marked increase in Src kinase activity during organogenesis. We can interpret the decrease in kinase activity in late embryonic stages as an effect of decreasing muscle activity, following the previous reports from chicken embryo, adult fish and frog muscles which show barely detectable kinase activity (22).

The expression of the Src family in the neural crest-derived tissues is both prominent and interesting. As well known, neural crest cells are extruded or migrated through dorsolateral pathway to the skin where they become the precursor of melanocytes, or through ventral pathway to a variety of locations where they become precursors of neuronal, endocrine and supportive tissues. Notably, all Src members appear in sequence in the neuronal tissues, such as spinal and cranial ganglia, and their nerve fibers (27). The immunoreactivity in the neural crest-derived non-neuronal tissues, including adrenal medulla, pigment cells, meninges, anterior ventral skull bones, and certain connective tissue of head and neck appeared later than that in the peripheral nervous system (PNS). Compared to the age related decline in Src immunoreactivity detected in the CNS, the cells of the PNS, endocrine and some other systems maintain high Src protein levels. Src expression in the neurons of the PNS which are capable of regeneration, and in the cells with high turnover rate implies that a Src protein in the brain and PNS is regulated differently. Besides, the adrenal medulla has a similar origin to the sympathetic nervous system and can be considered as a highly specialized adjunct of this system. As described above, Src expression appears restricted in specific population of cells. For example, our results point towards immunoreactivity in the adrenal medulla but not in the cortex of the adrenal gland. The findings suggest that Src expression is more crucial to the neuroectoderm-derived tissues than mesoderm origin.

As evidenced by the Src-immunostaining in the epithelium of the gastrointestine, tracheobronchial tree and urogenital duct, the thymus and liver are the other tissues which turnover fast and are highly immunostained. Additionally, Src expression was closely examined in the developing thymus, liver, CNS, retina, cartilage, bone, skin and limbs because cell death in these organs or tissues has been well documented (28). According to our results, the immunoreactivity in certain tissues appears to be limited at specific stages of development. For example, Src expression is quite strong in mesenchymal cells and cartilage cells early in cartilage formation, the period of cell death, but it is weaken in mature cartilage cells or undifferentiated mesenchymal cells. Similarly, in the mouse limb, some of the most prominent regions of cell death were the interdigital zones. Sre is strongly expressed in these zones around E14 and then maintains its presence in the cartilage and muscle tissues. Our results also indicated the presence of the Src family in the interdigital zones, suggesting that Src may be involved in the programmed cell death. In contrast, cells in the digits do not express Src and may be susceptible to avoid death. Although the role of preventing apoptosis for some Src members, $i$. e., Lyn and Fgr, during cellular differentiation has been studied (14), the precise function of the Src family regarding cell death is unclear. In addition, cell death occurs in all retinal cell types at various times. Our previous studies have observed that the distribution and time course of bcl-2 protooncogene protein, a regulator of apoptosis, and $\beta$-amyloid precursor protein, a precipitating factor in the pathological cascade of neurodegenerative diseases, expressed in the retinal ganglion cells coincides with a massive perinatal retinal death $(5,6)$. Likewise, the restrictive expression of the Src family in the inner layer of retinal ganglion cells suggests that its presence helps regulate the survival or death of retinal ganglion cells. Together, these findings suggest that cells displaying Src proteins may be classifiable as fast turnover or proliferating cells with rapid cell-death progression.

While most previous studies have revealed some members of the Src family expressed in the neurogenesis or organogenesis, suggesting their main functions were in regulating cell growth or differentiation (7), this study noted that Src family is present from the earliest cell cleavage periods, including the 2-cell to morula, blastocyst and two/three primary germ layer stages. During these early stages, embryo cells constantly divide, with cleavage creating future patterns and embyonic determination, suggesting that $\mathrm{Src}$ gene proteins are important in cell division. Our results greatly support the evidence that these tyrosine kinases play a role in growth, differentiation and maturation function, and suggest that the situation may be more complex than previously believed.

Herein, a genetic approach to the problem of 
overlapping function between Src kinase is adopted to dissect the signal transduction pathways and the critical role of various Src members. For instance, $s r^{c}$-null mice typically die within a few weeks of birth, while surviving mutant mice have impaired osteoclast function and develop osteopetrosis, and cell proliferation and other basal functions appeared unaltered. Moreover, no obvious abnormalities were noticed in the brain, suggesting the presence of other $s r c$-like kinase, $i$. e., c-fyn and/or c-yes, may impart a degree of functional redundancy with $c$-src (23). Besides, hematopoiesis appears to normal in the mutants with disruption of both $h c k$ and $f g r$, while Lyn protein activity is also increased in hck-null mice, indicating the deficiency of these two kinases is complemented by other Src members (17). Whereas previous studies have shown the genetic interactions between srC gene family members, our study provide further evidence that $\mathrm{Src}$ gene products have overlapping or complementary functions, particularly during embryogenesis in specific organs.

On the other hand, the variable granular intensity in the cytoplasm of early dividing cells and the consistent Src expression in the different germ layers from this study suggests that the members of Src family may function alternatively. The results also back up with previous reports that the $s r c$-related proteins (Fyn, Yes and Lyn) do not compensate for the absence of pp60- ${ }^{\text {c-src }}$ in the mutant mice, suggesting that the gene protein may have a specific function in osteoclasts and intracellular membranes $(13,23)$. Further work should examine the co-localization of the Src family in the developing tissues and organs of the normal and gene-disrupted embryos and to determine the role of Src members.

\section{Acknowledgments}

The authors would like to thank the National Science Council, Republic of China for financially supporting this under Contract No. NSC85-2331-B006-086.

\section{REFERENCES}

1. Appleby M. W., Gross J. A., Cooke M. P., Levin S. D., Qian X. and Perlmutter R. M. (1992) Defective T cell receptor signaling in mice lacking the thymic isoform of $\mathrm{p} 59^{\mathrm{ty}}$. Cell 70, 751-763.

2. Bolen J. B. (1993) Nonreceptor tyrosine protein kinase. Oncogene 8, 2025-2031.
3. Bolen J. B., Thompson P. A., Eiseman E, and Horak I. D. (1991) Expression and interactions of the Src family of tyrosine protein kinases in $\mathrm{T}$ lymphocytes. $A d v$. Cancer Res. 57, 103-149.

4. Brugge J. S., Cotton P. C., Queral A. E., Barrett J. N., Nonner D. and Keane R. W. (1985) Neurones express high levels of a structurally modified activated form of the $c-s r c$ gene product in neuronal cells. Genes Dev. 1, 287-296.

5. Chen S. T., Garey L. J. and Jen J. S. (1994) Bcl-2 protooncogene protein immunoreactivity in normal developing and axotomised rat retinas. Neurosci. Lett. 172, I 1-14.

6. Chen S. T., Patel A. J., Garey L. J. and Jen L. S. (1997) Expression of $\beta$-amyloid precursor protein immunoreactivity in the retina of the rat during normal development and after neonatal optic tract lesion. Neuroreport $\mathbf{8}$, 713-717.

7. Cooper J. A. and Howell B. (1993) The when and how of Src regulation. Cell 73, 1051-1054.

8. Courtneidge S. A., Fumagalli S., Koegl M., Superti-Furga G. and Twamley-Stein G. M. (1993) The Src family of protein tyrosine kinase: regulation and function. Development $\mathrm{S}, 57-64$.

9. Eiseman E. and Bolen J. B. (1990) src-related tyrosine protein kinases as signaling components in hematopoietic cells. Cancer Cells 2, 303-310.

10. Farrell J. E. and Martin G. S. (1988) Platelet tyrosinespecific protein phosphorylation is required by thrombin. Mol. Cell. Biol. 8, 3603-3610.

11. Gessler M. and Barnekow A. (1984) Differential expression of the cellular oncogenes $c$-src and $c$-yes in embryonal and adult chicken tissues. Biosci. Reports 4, 757770.

12. Hibbs M. L. and Dunn A. R. (1997) Lyn, a src-like tyrosine kinase. Int. J. Biochem. Cell Biol. 29, 397-400.

13. Horne W. C., Chatterjee N. D., Lomri A., Levy J. B. and Baron R. (1992) Osteoclasts express high levels of pp60 $0^{\text {c-src }}$ in association with intracellular membranes. $J$. Cell Biol. 119, 1003-1013.

14. Katagiri K., Yokoyama K. K., Yamamoto T., Omura S., Irie S. and Katagiri T. (1996) Lyn and Fgr proteintyrosine kinase prevent apoptosis during retinoic acidinduced granulocytic differentiation of HL-60 cells. $J$. Biol. Chem. 271, 11557-11562.

15. Kawakami T., Pennington C. Y. and Robbins K. C. (1986) Isolation and onco-genic potential of a novel human src-like gene. Mol. Cell. Biol. 6, 4195-4201.

16. Levy B. T., Sorge L. K., Meymandi A. and Maness P. F. (1984) pp60 c-src kinase is in chick and human embryonic tissues. Dev. Biol. 104, 9-17.

17. Lowell C. A., Soriano P. and Varmus H. E. (1994) Functional overlap in the $s r c$ gene family: inactivation of hck and $f g r$ impairs natural immunity. Genes Dev. 8, 387398.

18. Maness P. F. (1992) Nonreceptor protein tyrosine kinases associated with neuronal development. Dev. Neurosci. 14, 257-270.

19. Maness P. F., Sorge L. K. and Fults D. W. (1986) An early developmental phase of pp60 ${ }^{r-s r c}$ expression in the neural ectoderm. Dev. Biol. 117, 83-89.

20. Marth J. D., Disteche C., Pravcheva D., Ruddle F., Krebs E. G. and Perlmutter R. M. (1986) Localization of a lymphocyte-specific protein tyrosine kinase gene $(l c k)$ at a site of frequent chromosomal abnormalities in human lymphomas. Proc. Natl. Acad. Sci. USA 83, 7400-7404. 
21. PerImutter R. M., Marth J. D., Lewis D. B., Peet R. Ziegler S. F. and Wilson C. B. (1988) Structure and expression of $/ c k$ transcripts in human lymphoid cells. $J$. Cell. Biol. 38, 117-126.

22. Schart1 M. and Barnekow A. (1984) Differential expression of the cellular sic gene during vertebrate development. Dev. Biol. 105, 415 422.

23. Soriano P., Montogomery C., Geske R. and Bradley A. (1991) Targeted disruption of the c-src proto-oncogene leads to osteopetrosis in mice. Cell 64, 693-702.

24. Stein P., Lee H. M., Rich S. and Soriano P. (1994) pp59 $9^{m}$ mutant mice display differential signaling in thymocytes and peripheral T cells. Cell 70, 741-750.

25. Sukegawa J., Semba K., Yamanashi Y., Nishizawa M., Miyajima N., Yamamoto T. and Toyoshima K. (1987) Characterization of cDNA clones for the human $c$-yes gene. Mol. Cell. Biol. 7, 41-47.

26. Superti-Furga G. and Courtneidge S. A. (1995) Structurefunction relationships in Src family and related protein tyrosine kinases. Bioessays 17, 321-330.

27. Wang J. P., Chuang S. M., Shen C. L. and Chen S. T. (1998) expression of Src family during development of the mouse nervous system. Biomed. Res. 19, 315-325.

28. Webster D. A. and Gross J. (1970) Studies on possible mechanisms of programmed cell death in the chick embryo. Dev. Biol. 22, 157-184.

29. Yamanashi Y., Fukushige S., Semba K., Sukegawa J., Miyajima N., Matsubara K., Yamamoto T. and Toyoshima K. (1987) The yes-related cellular gene lym encodes a possible tyrosine kinase similar to p56 $6^{\text {ys }}$. Mol. Cell. Biol. 7, 237-243.

30. Yamanashi Y., Mori S., Yoshida M., Kishimoto T., Inoue K., Yamamoto T. and Toyoshima K. (1989) Selective expression of protein-tyrosine kinase, p56 $56^{1 \mathrm{yn}}$, in haematopoietic cells and association with production of human T-cell lymphotropic virus type I. Proc. Natl. Acad. Sci. USA 86, 6538-6542. 